\title{
Developing an Interface to Support Procedural Memory Training using a Participatory-Based Approach
}

\author{
Patrick Carrington ${ }^{1}$, Ravi Kuber ${ }^{1}$, Lisa Anthony ${ }^{1}$, Amy Hurst $^{1}$ and Sapna Prasad ${ }^{2}$ \\ ${ }^{1} \mathrm{UMBC}$ \\ ${ }^{2}$ Landmark College Institute for Research and Training \\ carpat1@umbc.edu
}

\begin{abstract}
For some individuals with executive function deficits, difficulties may be experienced when executing step-by-step procedures involving cognitive and motor skills. In this paper, we describe the design of a mobile application prototype, developed using a participatory-based approach, in order to enable individuals with executive function deficits to practice ordering steps within common-tasks. The long-term aim is to determine whether users are able to transfer the knowledge gained from using the application to the real world, in order to promote levels of independence. Lessons learned from conducting a participatory approach with individuals with executive function deficits are described.
\end{abstract}

Executive function deficits. Procedural memory. Participatory design.

\section{INTRODUCTION}

The ability to plan, organize and execute tasks is often taken for granted. However, for individuals with executive functioning deficits, difficulties experienced with procedural memory may directly impact the ability to perform self-care tasks or to live independently. Solutions, such as visual aids (e.g. planners, calendars, sticky notes, and checklists) and alert devices (e.g. watches with alarms) are often used as a strategy to support the planning or decision making process. While these are valuable tools, research indicates that rehabilitative exercises may help individuals with certain cognitive disabilities, to achieve the highest level of independence and quality of life possible (Wexner Medical Center (2011). For individuals with moderate to severe cognitive disabilities, the presence of a medical professional or caregiver may be required to assist with rehabilitation exercises. Existing research strongly suggests learning and memory as a general area to be supported through the use of technology (Hart et al. 2003). However, many rehabilitative interfaces are constrained to clinical settings or are expensive to purchase, making these difficult to obtain for home use.

Our research aims to identify ways to support individuals with deficits in procedural memory, through the development of a mobile rehabilitative interface. The goal is to assist the user by suggesting the steps taken to perform a task critical for independent living, and reinforce memorization of the process through rehearsal of steps. It is important for the user to recognize the necessary steps to perform the task, and the order that these belong in the sequence. The steps can then be applied to tasks within the user's own environment or an unfamiliar environment. The application can either be used independently or in conjunction with the user's caregiver, and can be used to supplement existing medical and rehabilitative interventions. In this paper, we describe the participatory design process, with a view to strengthening the design of the product.

\section{SUPPORTING PROCEDURAL MEMORY}

Interventions for strengthening procedural memory have often been performed through the use of cued recall. The primary rehabilitation area identified by Hart et al. (2003) was learning and memory, with planning and organization following closely behind. Although cued recall is not always explicitly stated as the intended goal or method, it is clear in several implementations that a similar approach has been adopted, whether through text reminders or images. Cued instructions were used in wayfinding experiments by Chang et al. $(2008 a, b)$, in order to familiarize their users with different landmarks or routes in order to improve their ability to 
independently navigate within their environment. Culley and Evans (2010) clearly state their method of cued recall to improve memory by using the SMS text feature common to most mobile computing devices. Participants in their study were reminded about their rehabilitation goals by daily text messages, with the aim of improving levels of activity.

Pagadala and Napper (1997) developed a computer-based cognitive aid that would allow patients to complete tasks almost independently, while still allowing their caregivers to monitor progress. Their system incorporated an adaptive interface that would display the steps in everyday tasks to the user. The steps in each task could be broken down into smaller tasks. The user would then interact with the system to indicate the completion of each task and subtask that was displayed to them. Over time, as the patients' ability and efficiency in completing the tasks improved, the steps would be consolidated. While the system was implemented using a desktop computer, a similar concept could be replicated using portable, commonly-owned technologies, such as cellular phones. Mobile applications could then be used irrespective of location. However, to develop an effective mobile solution, design would need to consider the unique requirements of individuals with executive functioning deficits.

\section{IMPORTANCE OF PARTICIPATORY-BASED APPROACHES IN INTERFACE DESIGN}

Participatory design is an approach to the design process that focuses on collaborating with intended users throughout the development lifecycle. Rather than simply asking the intended users for feedback and suggestions, users are considered members of the design team and work together with developers to create designs (Kuber, Yu \& McAllister 2007, Wu, Richards \& Baecker 2004). An iterative approach is adopted by default. Participatory approaches have been used to develop assistive technology prototypes, adhering to the needs of target groups such as older adults, and individuals with cognitive disabilities $[8,9]$. We have aimed to target the needs of individuals with attention and memory deficits. Examples include individuals with ADHD, who may experience problems retaining information in memory, due to inattentiveness or impairment in inhibiting environmental interference (Cox 2007). Wu, Richards, \& Baecker (2004) describe the importance of having multiple users with cognitive differences on a design team in order to improve the solutions that are created.

Our research aims to examine ways to support procedural memory among individuals with executive functioning deficits. In this paper, we describe the development of a mobile application prototype using a participatory-based approach, along with lessons learned from the process.

\section{DESIGN OF PROTOTYPE}

Through initial discussions with individuals with executive functioning deficits and medical professionals, a need was identified for a solution, simple in design, to rehearse common tasks essential to daily living (e.g. making a bed) which could be accessed on-the-fly, independent of location. A mobile prototype application was developed for the Android ${ }^{1}$ platform, using Applnventor. The application can be used independently or with the assistance of a caregiver. The application can be customized to meet the needs of the user, enabling him/her to rehearse tasks that are essential to improving the user's personal quality of life.

The prototype system presented here consists of three main interfaces: one that would be used to enter the steps in the processes to be learned, one to actually learn the procedure, and the third to practice putting the steps of the procedure in order.

\subsection{Interface 1: Adding a Procedure}

The first interface is intended to provide the user or a caregiver with a way to add new activities to the application, and specify the number of steps that are required to complete that activity. This allows the application to be adaptive in that the caregiver and user may customize the available activities to their unique situation.

The interface consists of textboxes where the task name and steps involved in the task can be added. Each step is associated with an image, which can be uploaded in the same manner. The activity name and steps to perform the procedure are stored in a database local to the mobile device.

\subsection{Interface 2: Procedure Trainer}

The second interface consists of 3 screens: a) the menu, b) the Activity Viewer, and c) the quiz (described in a later section). From the menu users may enter the name of the procedure/activity they wish to learn or practice, then push a button to launch the viewer. The activity viewer (Figure 1 left) provides the user with an interface to learn the steps associated with the activity. This interface consists of 3 main areas. The step number and text description are displayed at the top of the screen. Directly below that are three buttons that allow the user to navigate through the steps in the procedure. They have the option of stepping

\footnotetext{
${ }^{1}$ Android - http://www.android.com
} 
through the activity manually, or by pressing the play button, which will automatically cycle through the steps. This process can be repeated as many times as needed by the user. The goal of the interface is to familiarize the user with the ordering of the steps in the procedure. Additionally, displaying graphical cues should assist the user to remember the instructions for each step. As each step is displayed, a corresponding textual description associated with each image is read aloud using the text-to-speech component in the SDK. The user or caregiver can launch the quiz at any time, via the button underneath the viewing area.
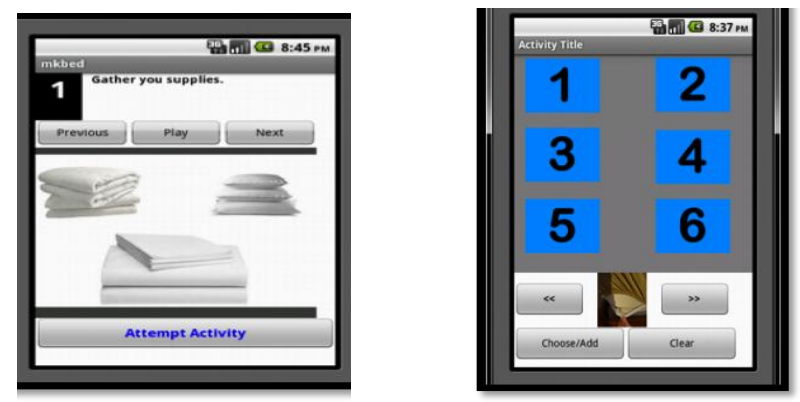

Figure 1: The Procedure Trainer Activity Viewer (left) and Quiz interface (right).

By enabling the user to modify the pace in which he/she views the steps in each activity, it is possible for him/her to focus on each step according to their individual needs. Some users may need to spend more time examining specific steps in detail, while others may need to cycle through the entire sequence quickly, repeatedly, to commit the order to memory.

\subsection{Interface 3: Quiz Interface}

The quiz interface is shown in Figure 1 (right). The purpose of the quiz interface is to give the user the opportunity to arrange the steps of the procedure in order, after having viewed all of the steps associated with the procedure.

The following brief description of this interface is intended to give the reader a general idea of what we were attempting to design. The user is able to view images of each step in a randomized order. $\mathrm{He} / \mathrm{she}$ is prompted to arrange these images in the order of the task. Once the last image has been selected and positioned within the sequence, a submit button appears on the screen, which the user is able to select. Upon submission, the images in the display area are replaced by the word "Done", in each image slot, to provide feedback to the user of the action performed. The user is then given feedback on their performance.

\section{PARTICIPATORY DESIGN WORKSHOP}

The workshop took place at a two-year college which caters specifically to the needs of students with learning differences, encompassing attention deficit hyperactivity disorder (ADHD), autism spectrum disorders (ASD) and EFD (Anthony et al. 2012). The following section describes the format of the participatory design workshop as well as briefly discussing the modifications that resulted from each design session.

The first session involved three students (aged 1829) who self-identified as experiencing learning differences. One researcher, with interface design experience, participated in the group. Participants were presented with a basic introduction to the application by the researcher. They were asked to freely explore the application, and offer suggestions for improvement. The participants were observed discussing the utility of the application, the types of tasks that could be rehearsed which would be useful for independent living, and how the application could be targeted to relate to their own needs (e.g. completing the steps associated with math problems). While the design of the application was thought to be functional, participants suggested that users would need to be motivated to rehearse tasks.

The group suggested moving away from a quizstyle application, and making the interface into a fun, yet challenging game. The students suggested games similar to card matching as well as other types of quizzes (multiple choice, fill in the blank, true or false, etc.), which were represented diagrammatically. Building on their ideas, students were queried on other ways to motivate target users. Suggestions included integrating reward systems (e.g. earning points/tokens) and the ability to track progress (e.g. displaying scores). Participants suggested the ability to share performance information with friends, or just other users. However, after further discussion, it was found that it would not be feasible to compare scores with one another, as the settings for each game would be tailored to the needs of individuals with varying levels of ability.

Participants were asked to rework their original designs integrating the items discussed within the session. These diagrams would be shown to the next group.

The second group consisted of 3 students (aged 18-29) who had not previously accessed the prototype. One researcher with interface design experience also participated in this group. The participants were introduced to the prototype and diagrams developed by the first group. They were able to offer fresh perspectives on iterating the 
existing interface. Examples included providing concise instructions, to ensure that the user would stay on track, and offering rewards based on performance. Participants preferred to externalize their representations diagrammatically, as it was found to be easier to convey among others in the group. Towards the end of the session, participants were able to select more suitable designs targeted to the needs of individuals with executive functioning deficits, from the range of diagrams created, and discuss the challenges which may be encountered when accessing these types of solutions.

On occasion, individuals in the group would need additional guidance to perform the task. For example, even though the group were asked to diagrammatically represent their ideas, one participant insisted on writing actual Java code to develop the game, rather than sketching a simple wireframe of how the interface should appear. Each participant was found to approach problems in a different way, and expressed different levels of concern regarding the level of detail in their solutions.

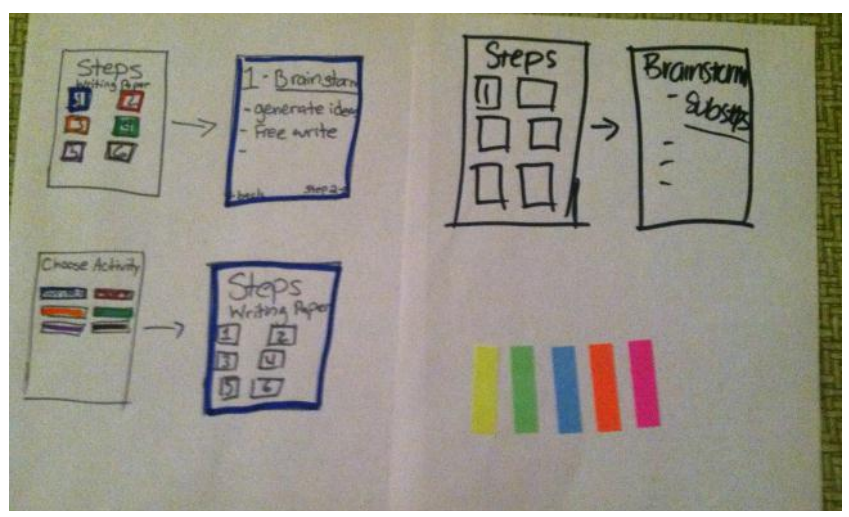

Figure 2: Example of a drawing from the second design session displaying colour options for navigation.

During this session the students pointed out that with the existing interface, it would be difficult to display procedures that had more than six steps or that contained sub-steps, due to the limited screen size of the mobile device. The interface designer in the group was able to offer advice on ways in which navigation could be designed to support the user, from their own experience of designing quiz-style applications. These suggestions could be strengthened by the students (Figure 2). The team worked together to try to identify how to best track and display performance over time, as suggested during the earlier session. The group reached consensus that line graphs would be the most effective method of identifying progress. However, these should be customized to show specific periods of time, with options that could be selected and deselected to customize the amount of information that the user sees with a view to reducing overload.

The group suggested that a reworked version of the application should log the following items: 1) number of times a quiz was completed, 2) number of attempts until an accurate attempt was made, 3) number of accurate attempts, 4) time taken to perform the task. This information could be used for scoring purposes.

The final item discussed during the session was the name of the application. It was determined that in order to get people to even consider choosing this application or to remember it, a catchy name would be necessary. "ProcedurePal" was decided upon.

The students in both sessions were very willing to provide feedback and ideas. They were especially interested in talking about whether or not it could be useful for themselves, or for their peers with similar learning differences. They also suggested that the application could be used while performing a real world task, to provide prompts to perform particular steps. For example, when the user views the image of plumping a pillow on the mobile interface, he/she could replicate the action when making his/her bed in his/her student residence.

The following section describes the lessons learned from the participatory-based approach undertaken, to develop a mobile interface to support individuals with executive functioning deficits.

\section{DISCUSSION OF PARTICIPATORY-BASED APPROACH}

\subsection{Images to Support Discussion}

The use of graphical stimuli (e.g. images of the interface) appeared to significantly increase the productivity of the participants during the design sessions. At the beginning of the first session, the students were given an oral introduction to the application. At this point, only one participant was able to offer ideas regarding strengthening the design of the solution. Screenshots of the existing interface were then handed out. Communication was immediately initiated. The students began to ask questions about the design of the interface and voice their opinions regarding ways in which the solution could be improved. Participants were also observed pointing to features on the screenshots when describing design ideas. This helped the remainder of the group to follow along, and critique the ideas generated.

\subsection{Use of Diagrams to Focus Attention}

During the sessions, students with learning differences were sometimes observed to 
experience difficulties focusing upon the task-inhand. This seemed to occur especially when discussing the critiques of different design options. Our approach to this issue was to encourage the participants to either externally represent their own design idea, or to work together with the remainder of the group to build an image of the interface. This helped all of the students to focus their attention on the current task and not on what was going on in other areas of the room. They could then critique and annotate one another's designs.

\subsection{Importance of Group to Trigger Ideas}

By working together as a group, students were observed to build upon one another's design ideas. For example, one participant was unable to identify a solution for the current prototype's inability to handle sub-steps effectively. When the issue was presented to the group, it was suggested that colouring or numbering the steps differently may aid target users. This suggestion triggered a longer discussion of different ways to show sub-steps using colours and numbers. Had the group not been able to weigh in on these design options, a solution may not have been suggested. Additionally, the ideas expressed by the group in terms of how the sub-steps should be presented, arose from the experiences of the participants when accessing their favourite quiz-style applications, thus increasing the likelihood that the chosen solution would be effective.

\subsection{Application of the Participatory Design Approach}

In contrast with user-centred design approaches, participants were able to assume an active role in the design process for the application. Encouraging participants to weigh in as designers of the application increased their feeling of ownership to the application's success. This is similar to findings by Ellis and Kurniawan [9], regarding the participants viewing the design sessions as a service to other similar users. Participants were able to discuss different design ideas amongst themselves and provide an insight to their own experiences. This led to the development of ideas that were more applicable to real-world scenarios, which individuals with executive functioning deficits may face.

Some of the drawbacks to participatory design with this group in particular stem from difficulties with attention and focus. It was increasingly difficult to keep everyone in the group focused on a single task for long periods of time. This caused challenges when it came time to discuss different design options. While the use of graphical stimuli (e.g. images and diagrams) helped to refocus the group, this still affected the productivity of the sessions, especially as time was a limited resource. Another challenge faced when adopting a participatory design approach is that each participant may have his or her own way of solving problems that are not conducive to supporting discussion among the group. In these cases, it is important to recognize these differences and attempt to find a common ground or medium that allows for the sharing of ideas with the whole group and not just with one or two individuals.

\section{CONCLUSION AND FUTURE WORK}

In this paper, a participatory-based approach was adopted to develop a mobile application prototype, addressing the needs of individuals with executive functioning deficits. Participants provided valuable feedback and presented a range of new design ideas for improving the usability of the application. Participants demonstrated the ability to build-upon each other's ideas, taking an active role in the design process.

The next logical step for the research would be to integrate findings from the workshop with the prototype, and perform a longitudinal study to monitor the efficacy of the application. We also aim to determine whether individuals with executive functioning deficits are able to transfer knowledge gained from using the application, to apply to the real world (e.g. to make their own beds using the same order of steps suggested in the application). The aim would be to provide support the user to perform tasks which would aid independent living.

\section{ACKNOWLEDGEMENTS}

The authors would like to thank Michelle Bower, Geoff Burgess, Barbara Linam-Church, Kirk Norman and Flynn Wolf for their support running the participatory design workshop. The participatory design workshop was funded by the Alliance for Access to Computing Careers (AccessComputing)

http://www.washington.edu/accesscomputing/

\section{REFERENCES}

Anthony, L., Prasad, S., Hurst, A. and Kuber, R. (2012) A participatory design workshop on accessible apps and games with students with learning differences. In: Proceedings of ASSETS'12. Boulder. 22-24 October 2012. ACM Press.

Chang, Y.J., Chen, C.N., Chou, L.D. and Wang, T.Y. (2008a) A novel indoor wayfinding system based on passive RFID for individuals with cognitive impairments. In: Proceedings of 
Pervasive Computing Technologies for Healthcare, Tampere. 30 January-1 February 2008. IEEE. 108111.

Chang,,Y., Chu, Y., Chen, C. and Wang, T. (2008b) Mobile computing for indoor wayfinding based on Bluetooth sensors for individuals with cognitive impairments. In: Proceedings of the International Symposium on Pervasive Computing, Santorini. 79 May 2008. IEEE. 623-627.

Cox, A.J. (2007) Executive function and ADHD: Overview and intervention strategies for parents and teachers. Lehigh Psychological Services. Available from http://www.lehighpsych.com/art_adhd.htm (1 May 2012).

Culley, C. and Evans, J.J. (2010) SMS text messaging as a means of increasing recall of therapy goals in brain injury rehabilitation: a singleblind within-subjects trial. Neuropsychol Rehab, 20 (1), 103-119.

Ellis, R.D. and Kurniawan, S. (2000) Increasing the usability of online information for older users: a case study in participatory design. Intern. J. Human-Computer Interaction, 12 (2), 263-276.
Hart, T., O'Neil-Pirozzi, T. and Morita, C. (2003) Clinician expectations for portable electronic devices as cognitive-behavioural orthoses in traumatic brain injury rehabilitation. Brain Inj., 17 (5). 401-411.

Kuber, R., Yu, W. and McAllister, G. (2007). Towards developing assistive haptic feedback for visually impaired internet users. In: Proceedings of CHI'07, San Jose. 30 April - 3 May 2007. ACM Press. 1525-1534.

Pagadala, R. and Napper, S. (1997) An adaptive cognitive orthosis for task guidance. In: Proceedings of Biomedical Engineering Conference, Biloxi. 4-6 April 1997. IEEE. 422-424.

Wexner Medical Center (2011) Rehabilitation. Ohio State University. Available from http://medicalcenter.osu.edu/ patientcare/healthcare_services/stroke/rehab May 2012).

Wu, M., Richards, B. and Baecker, R. (2004). Participatory design with individuals who have amnesia. In; Artful Integration: Interweaving Media, Materials and Practices. Proceedings of Participatory Design Conference'04, Toronto. 27-31 July 2004. ACM Press. 214-223. 